\title{
A giant black hole at the centre of a dwarf galaxy
}

\section{from C. Martin Gaskell}

THE dwarf elliptical galaxy M32, one of the satellite galaxies of the nearby spiral galaxy M31, has recently been shown to have a central dark object with a mass five million times greater than the Sun ${ }^{1}$. This is the most convincing case yet of a massive black hole in the centre of a galaxy.

Signs of violent activity are to be found in the nuclei of many galaxies. They range from modest goings-on in our own Galaxy to the tremendous outpourings of energy in quasars. The source of this energy is believed to be the accretion of matter onto massive black holes ${ }^{2,3}$ but the evidence is still only indirect. One obvious way to detect a massive black hole is through its gravitational attraction on nearby stars. In 1978, the late Peter J. Young and his collaborators $\mathrm{s}^{4,5}$ claimed to have found just such an effect; in the very centre of the giant elliptical galaxy M87, the average speed of the stars shoots up dramatically, as though by sudden strong gravitational pull. M87 is certainly a galaxy in which one would expect a massive black hole because it is relatively active, but the claim soon came under attack. Theoretical studies ${ }^{6-8}$ showed that the observations of Young and his collaborators ${ }^{4,5}$ could be explained without invoking the presence of a black hole (of $5 \times 10^{9}$ solar mass) if the orbits of the stars were predominantly radial (rather than random). Further observations ${ }^{9}$ showed a dense concentration of stars in the centre of M87, which also had to be taken into consideration. The presence of a black hole in M87 remains possible but is certainly not necessary.

M32 has perhaps a thousand times fewer stars than a giant elliptical galaxy like M87 and is certainly not an active galaxy. It does, however, show an unexplained concentration of stars towards its centre. Using the 200-inch telescope on Mt Palomar to study the motions of the stars in and near this central concentration, John Tonry has now found that, as with M87, the dispersion in the velocities of the stars rises suddenly towards the centre. But unlike M87, the system of stars as a whole is clearly rotating in $\mathrm{M} 32$, so radial orbits

I. Tonry J.L. Atrophys, J. Lett. 283, L27 (1984).

2. Zeldovich, Y.B. \& Novikov, 1.D. Dokl. Acad. Nauk. SSSR 158, 811 (1964)

3. Salpeter, E.E. Astrophys, J. 140, 796 (1964).

4. Young, P.J., Westpal, J.A., Kristian, J. \& Wilson, C.P. Astrophys. J. 221, 721 (1978).

5. Sargent, W.L.W., Young, P.J., Boksenberg, A., Shortridge, K., Lynds, C.R.\& Hardwick, F.D.A. Astrophys, J., 221, 731

6. Duncan, M.J.\& Wheeler, J.C. Astrophys. J. Lett. 237, L27 (1980)

7. Binney, J.\& Mamon, G.A. Mon. Not. R. astr. Soc. 200, 361 (1982)

8. Tonry, 3. A. Atrophys. J. 266, 58 (1983)

9. Dressler, A. Astrophys. J. Lett. 240, LII (1980). cannot be the explanation; there has to be some excess mass there. Moreover, this mass is more than can readily be accounted for by the stars alone; an extra five million times the mass of our Sun is present, according to Tonry. A massive black hole is the most likely explanation.

If there is a black hole of such size in the middle of M32, why is the galaxy totally inactive? The answer is probably that there is nothing to fuel the black hole; gas is escaping from the galaxy rather than falling into the centre, as it would in a more massive galaxy.

M32 is by no means unique in having a dense central condensation of stars. It just happens to be very near and is therefore easy to study. To make comparable observations on more distant examples will need better resolution than can be achieved from the ground. When the Space Telescope (due to be launched towards the end of next year) turns on the nuclei of these galaxies I, for one, am pretty confident that they too will show signs of massive objects lurking in their centres.

C.M. Gaskell is in the Astronomy Department, University of Texas, Austin, Texas 78712, USA.

\section{Vision}

\section{Fireworks in the retina}

\section{from David I. Vaney}

VISUAL information undergoes a sophisticated coding process in the retina, culminating in the diverse and functionally complex output of the ganglion cells to the brain: The receptive fields of the ganglion cells are shaped by the preceding interneurones, which include a set of neurones without axons, termed amacrine cells. The responses of amacrine cells are poorly documented because it is difficult to record from their small cell bodies, and the responses obtained may not reflect the local interactions between adjacent input and output synapses on the amacrine branches. In a recent series of elegant papers, however, R.H. Masland and his colleagues elucidate the functional properties of one small population of amacrines, those that contain acetylcholine ${ }^{1}$. Their findings suggest that the plexus formed by the branches of these cells provides the substrate for motion detection within subunits of a ganglion cell's receptive field.

In the rabbit retina, about four per cent of the amacrine cells synthesize and release acetylcholine. Their cell bodies are symmetrically distributed on both margins of the inner plexiform layer (IPL, the narrow zone containing processes of ganglion cells and presynaptic interneurones) and their terminals form two dense bands within the IPL ${ }^{4,5}$. These cells are of particular interest to retinal physiologists because they are implicated in the generation of direction selectivity in two types of ganglion cells ${ }^{6}$. Masland et al. have neatly side-stepped the problems of electrophysiological recording by studying the morphological characteristics and pharmacological responses of the whole population of these cells, using a sophisticated range of techniques. Although the shape and distribution of these neurones were presaged by previous studies $^{7-10}$ which used classical histological methods, this integrated approach provides definitive descriptions and fresh insights. Masland, Mills and Hayden ${ }^{1}$ have shown that the acetylcholine-synthesizing amacrine cells identified by autoradiography are also selectively labelled in vivo by the fluorescent dye diamidino-phenylindole (DAPI), so that the cells can be identified and mapped in a whole-mounted retina. Tauchi and Masland ${ }^{2}$ have impaled the DAPI-labelled cells with a micropipette under visual control and iontophoretically injected the fluorescent dye Lucifer yellow into the cytoplasm. This reveals a characteristic dendritic morphology that has been compared to a 'starburst' firework ${ }^{9}$, and confirms the previous description by Vaney using similar methods ${ }^{11}$ (Fig.1). The arborization ranges from $200-800 \mu \mathrm{m}$ in diameter but is virtually planar, with the distal dendrites narrowly stratified in one of the two bands in the plexiform layer that contain acetylcholine.

Masland, Mills and Cassidy ${ }^{3}$ have demonstrated that the two morphological subtypes of cholinergic amacrines respond differently to visual stimuli; those branching in the inner half (on the ganglioncell side) of the plexiform layer release a transient burst of acetylcholine at light $\mathrm{ON}$, whereas those with branches in the outer half of the layer release a burst at light OFF (Fig.2). This has been established convincingly by monitoring the distribution and release of labelled acetylcholine in response to visual stimulation in vitro, while using a pharmacological agent known to block the transmission of $\mathrm{ON}$ responses.

The most striking feature of the cholinergic amacrine cells is the extensive overlap of their dendrites in the plexiform layer: each point on the retina is covered by the dendritic trees of 25-70 cells of each subtype ${ }^{2,11}$, an order of magnitude greater than the overlap of the horizontal or ganglion cell arborizations in the cat retina ${ }^{12}$. 